\title{
Strates
}

STRATES Matériaux pour la recherche en sciences sociales

Hors-série | 2002

Parcours dans la recherche urbaine, Michel Rochefort, un géographe engagé

\section{Un directeur de labo pas comme les autres}

\section{Raymonde Ladefroux}

\section{(2) OpenEdition}

12 Journals

Édition électronique

URL : http://journals.openedition.org/strates/526

DOI : $10.4000 /$ strates.526

ISSN : 1777-5442

Éditeur

Laboratoire Ladyss

Édition imprimée

Date de publication : 1 janvier 2002

ISSN : 0768-8067

Référence électronique

Raymonde Ladefroux, "Un directeur de labo pas comme les autres », Strates [En ligne], Hors-série

2002, mis en ligne le 02 mai 2005, consulté le 08 septembre 2020. URL : http://

journals.openedition.org/strates/526 ; DOI : https://doi.org/10.4000/strates.526

Ce document a été généré automatiquement le 8 septembre 2020

Tous droits réservés 


\title{
Un directeur de labo pas comme les autres
}

\author{
Raymonde Ladefroux
}

1 Quand je pense à mes débuts à l'Institut de géographie, je revis ce jour de février 1967 qui marqua pour moi le commencement d'une vie professionnelle enfin riche et épanouissante.

2 Je me revois attendant avec anxiété dans le couloir du premier étage de l'Institut de géographie d'être reçue par monsieur Michel Rochefort en vue d'une nouvelle affectation au Cnrs, à la suite d'une demande de changement de service consécutive à une expérience professionnelle traumatisante. Une porte s'ouvre, une voix éclatante m'appelle, et un monsieur grand, au visage barbu et jovial, m'invite à entrer dans un bureau d'une austérité surprenante, accoutumée que je suis à l'élégance confortable des bureaux de direction du quai Anatole France. Monsieur Rochefort m'explique que vient de se créer une « section de Géographie humaine appliquée » rattachée au Centre de documentation de l'Institut de géographie et dont il est le responsable. Il cherche donc une personne pour commencer à structurer cette section. La conversation s'engage sur un ton si cordial que je sens peu à peu diminuer la timidité paralysante qui a toujours été la mienne dans mes relations de travail; je sors satisfaite de cet entretien, étant admise à commencer mon travail dès la semaine suivante.

Mon « installation » constitue pour moi une vraie surprise : pas de bureau à l'Institut, mais monsieur Rochefort me conduit en voiture dans une petite rue du quatorzième arrondissement, la rue Montbrun, et à mon grand étonnement, se dirige vers une boutique portant au sommet de sa devanture un calicot blanc, où s'inscrit "Électronique en haute fidélité ». Le rideau de fer ayant été relevé, je suis invitée à entrer dans la "boutique» aux murs tendus d'un tissus écossais à dominante verte, meublée d'un bureau, d'une petite étagère, d'une table sur laquelle sont posés une machine à écrire et un téléphone, et d'un radiateur électrique. J'en reçois les clés qui me permettront, pendant plus de dix-huit mois, de monter chaque matin, et de baisser chaque soir, le rideau de fer... Et bien souvent durant toute cette période, j'ai vu entrer 
dans mon «magasin» des personnes venues me demander des informations sur la haute fidélité...

4 Je me souviens aussi de mon tout premier travail : une très longue liste de statistiques, dont je dois tirer des pourcentages. Je m'y attaque, poursuivant chez moi les calculs très tard le soir, dans la crainte de ne pas avoir terminé ce travail quand le "patron » doit revenir à la "boutique », trois jours après mon installation (sans calculatrice, machine encore onéreuse à l'époque, je fais toutes les règles de trois sur papier...). Le terme fatal arrivant sans que la tâche soit achevée, je m'attends à être blâmée et jugée incapable. C'est un grand éclat de rire qui répond à mes explications désolées : « Mais je ne comptais pas sur l'achèvement de ce travail pour aujourd'hui, c'est un travail complémentaire aux activités que je vais vous donner dès aujourd'hui; vous l'achèverez donc au fur et à mesure de vos disponibilités. »

5 Et ainsi, je découvre jour après jour une relation professionnelle agréable et confiante. Ma formation première n'étant pas géographique, je demande à monsieur Rochefort de m'informer au moins sur les possibilités d'une initiation minimale en ce domaine : sans hésiter, il me met sur la voie d'une formation géographique complète. En outre, grâce aux travaux qu'il me confie, il m'ouvre les portes du Brésil qui tiendra plus tard une si grande part dans ma vie professionnelle, et même dans ma vie privée, vu les liens amicaux que je continue à cultiver dans ce pays.

De temps en temps, l'équipe de recherches dirigée par monsieur Coquery vient tenir des réunions dans la «boutique ». C'est là que je commence à faire connaissance avec deux de mes futures collègues, vacataires à l'époque, mais qui sont à l'heure où sont écrites ces lignes des chercheurs essentiels au sein du laboratoire Ladyss ${ }^{1}$.

7 En septembre 1968, je quitte la rue Montbrun pour un bureau de l'Institut de géographie ; la « section de Géographie humaine appliquée » devient «Laboratoire de géographie humaine ", laboratoire du Cnrs associé à l'université de Paris 1, avec à sa tête Michel Rochefort. Je vois avec plaisir l'arrivée au sein de la nouvelle formation de plusieurs collègues, et si les locaux, une seule pièce où sont entassés trois bureaux, une grande table, une photocopieuse et des étagères, deviennent de plus en plus étroits, l'ambiance y est des plus chaleureuses.

8 Il règne souvent dans ces locaux étriqués une atmosphère de ruche, notamment quand il s'agit de "boucler " un contrat de recherche ou, tous les deux ans, les volumineux rapports de fin d'exercice qui doivent être envoyés à l'administration centrale du Cnrs. À ces échéances, au travail intellectuel intense de nombreux mois, fait suite, pour tout le personnel Cnrs, durant des jours jusqu'à l'heure de fermeture de l'Institut, une activité fébrile pour achever de dactylographier, ronéoter, paginer, relier des documents qui doivent être remis à une date impérative. C'est qu'en effet, en l'absence d'un personnel de secrétariat sans cesse demandé, mais toujours refusé, ce sont tous les membres du laboratoire qui doivent s'atteler à ces tâches. Nombreux sont les étudiants qui passent par la salle du laboratoire en attendant d'être reçus par Michel Rocherfort dont le bureau se trouve dans la pièce attenante. Leur attente n'est pas monotone, car ils participent à l'atmosphère cordiale et joyeuse qui est celle, notamment, de la première moitié des années 1970 ; aux facéties d'un vacataire désopilant et doué en outre pour le dessin humoristique, répondent immédiatement les chansons de Jeanine, dont le répertoire inépuisable est capable de s'adapter à toutes les situations... Parfois même, le directeur y mêle sa voix puissante, en déclamant quelques vers de circonstances... Au cours de ces années, les équipes de recherches vont aller en se 
multipliant et en s'étoffant, regroupant chercheurs Cnrs et enseignants-chercheurs. Dans les années 1970, le laboratoire est donc une formation pleine de jeunesse, où le sérieux du travail n'interdit pas une joie de vivre à laquelle contribue son directeur, grâce à son humour et son sens humain.

Enfin, dans le contexte général plus difficile des années 1980, c'est encore sa compréhension humaine et sa diplomatie qui contribuent à aplanir certaines tensions et à maintenir la cohésion d'un laboratoire de plus en plus étoffé et où les restrictions budgétaires sont souvent pesantes, avant la nouvelle évolution qui aboutit à "Strates ". Malgré ou à cause de la reconnaissance que je lui porte, j'ai toujours résisté à une demande réitérée de Michel Rochefort : celle de l'appeler par son prénom. Aujourd'hui, en hommage affectueux, je vais adapter la réponse de Farah à Karen Blixen, concluant le film Out of Africa : «Vous êtes Michel, Monsieur ».

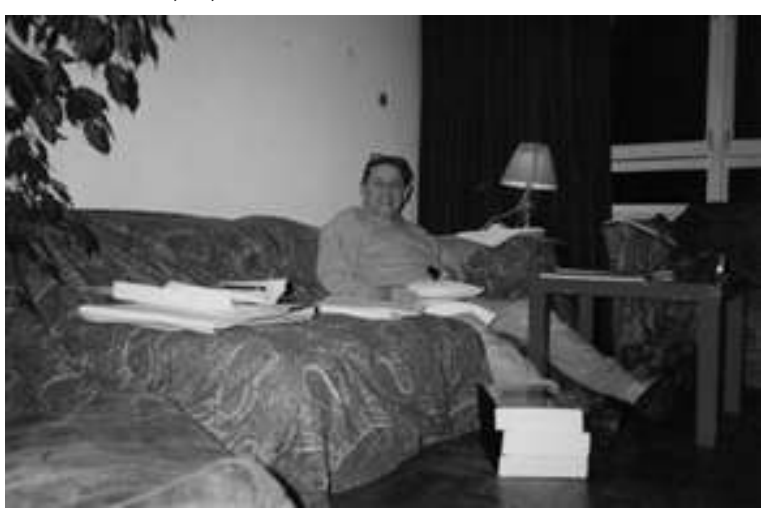

Michel Rochefort élaborant chez lui un rapport de thèse, Paris (1990)

\section{NOTES}

1. Laboratoire Ladyss dont l'une des composantes est le Laboratoire de géographie «Strates », lui-même issu du Laboratoire de géographie humaine (cf. infra).

\section{AUTEUR}

\section{RAYMONDE LADEFROUX}

Strates, Cnrs. 